\title{
Carcinoembryonic Antigen Improves the Performance of Magnetic Resonance Imaging in the Prediction of Pathologic Response after Neoadjuvant Chemoradiation for Patients with Rectal Cancer
}

\section{Gyu Sang Yoo, MD \\ Hee Chul Park, MD, PhD' \\ Jeong II Yu, MD, PhD' \\ Doo Ho Choi, MD, PhD' \\ Won Kyung Cho, MD \\ Young Suk Park, MD, PhD² \\ Joon Oh Park, MD, PhD² \\ Ho Yeong Lim, MD, PhD² \\ Won Ki Kang, MD, PhD'2 \\ Woo Yong Lee, MD, PhD 3 \\ Hee Cheol Kim, MD, PhD ${ }^{3}$ \\ Seong Hyeon Yun, MD, $\mathrm{PhD}^{3}$ \\ Yong Beom Cho, MD, $\mathrm{PhD}^{3}$ \\ Yoon Ah Park, MD, PhD ${ }^{3}$ \\ Kyoung Doo Song, MD, PhD ${ }^{4}$ \\ Seok-Hyung Kim, MD, PhD ${ }^{5}$ \\ Sang Yun $\mathrm{Ha}, \mathrm{MD}, \mathrm{PhD}^{5}$}

${ }^{1}$ Department of Radiation Oncology,

${ }^{2}$ Division of Hematology-Oncology,

Internal Medicine, Departments of

${ }^{3}$ General Surgery, ${ }^{4}$ Radiology, and ${ }^{5}$ Pathology,

Samsung Medical Center, Sungkyunkwan

University School of Medicine, Seoul, Korea

Correspondence: Jeong Il Yu, MD, PhD

Department of Radiation Oncology,

Samsung Medical Center, Sungkyunkwan

University School of Medicine, 81 Irwon-ro,

Gangnam-gu, Seoul 06351, Korea

Tel: $82-2-3410-2438$

Fax: 82-2-3410-2619

E-mail: jeongil.yu@samsung.com

Co-correspondence: Kyoung Doo Song, MD, PhD Department of Radiology, Samsung Medical

Center, Sungkyunkwan University School of

Medicine, 81 Irwon-ro, Gangnam-gu,

Seoul 06351, Korea

Tel: 82-2-3410-0518

Fax: 82-2-3410-2619

E-mail: kd3893.song@samsung.com

Received May 13, 2019

Accepted September 24, 2019

Published Online September 25, 2019

*Gyu Sang Yoo and Hee Chul Park contributed equally to this work.

\section{Purpose}

The purpose of this study was to investigate the role of carcinoembryonic antigen (CEA) levels in improving the performance of magnetic resonance imaging (MRI) for the prediction of pathologic response after the neoadjuvant chemoradiation (NCRT) for patients with rectal cancer.

\section{Materials and Methods}

We retrospectively reviewed the medical records of 524 rectal cancer patients who underwent NCRT and total mesorectal excision between January 2009 and December 2014. The performances of MRI with or without CEA parameters (initial CEA and CEA dynamics) for prediction of pathologic tumor response grade (pTRG) were compared by receiver-operating characteristic analysis with DeLong's method. Cox regression was used to identify the independent factors associated to pTRG and disease-free survival (DFS) after NCRT.

\section{Results}

The median follow-up was 64.0 months (range, 3.0 to 113.0 months). On multivariate analysis, poor tumor regression grade on MRI (mrTRG; $p<0.001)$, initial CEA $(p<0.001)$ and the mesorectal fascia involvement on MRI before NCRT (mrMFI; $p=0.054$ ) showed association with poor pTRG. The mrTRG plus CEA parameters showed significantly improved performances in the prediction of pTRG than mrTRG alone. All of mrTRG, MrMFI, and initial CEA were also identified as independent factors associated with DFS. The initial CEA further discriminated DFS in the subgroups with good mrTRG or that without mrMFI.

\section{Conclusion}

The CEA parameters significantly improved the performance of MRI in the prediction of pTRG after NCRT for patients with rectal cancer. The DFS was further discriminated by initial CEA level in the groups with favorable MRI parameters.

\section{Key words}

Rectal neoplasms, Neoadjuvant, Chemoradiation, Carcinoembryonic antigen, Magnetic resonance imaging, Pathology, Response, Disease-free survival 


\section{Introduction}

Neoadjuvant chemoradiotherapy (NCRT) followed by total mesorectal excision (TME) has been the mainstay of treatment for locally advanced rectal cancer since the German Rectal Cancer Study Group reported the superiority of NCRT to adjuvant chemoradiotherapy $[1,2]$. NCRT also allows the prediction of disease progression after surgery by its pathologic response [3-5]. In particular, pathologic complete response (pCR) after NCRT is a well-known prognosticator associated with improved disease-free survival (DFS) $[5,6]$. However, there is concern about some complications, such as low anterior resection syndrome and sexual dysfunction, after pelvic irradiation and radical surgery [7-9]. Therefore, the watch-and-wait strategy after NCRT with omission of surgery has been proposed in selected populations [10-13]. To forgo surgery safely, it is essential to identify the patients who would achieve pCR before surgery $[10,14]$.

Various diagnostic tools including physical examination, endoscopy, imaging, and tumor markers for the prediction of pCR have been investigated. Among those tools, the response on magnetic resonance imaging (MRI) after NCRT has shown correlation with pathologic responses and prognosis of patients with rectal cancer $[15,16]$. However, MRI alone is not a reliable tool for the prediction of pathologic response. Carcinoembryonic antigen (CEA) levels also show some correlations with pathologic response; however, this is not an absolute predictor [17-19]. To improve the performance in the prediction of pathologic response, a combination of multiple parameters is considerable, and there have been no relevant studies.

In this study, we aimed to investigate the role of CEA parameters in improving the performance of MRI in the prediction of pathologic response after NCRT for patients with rectal cancer primarily, and the relevant DFS, secondly.

\section{Materials and Methods}

\section{Patients}

We retrospectively reviewed the medical records of 764 rectal cancer patients who underwent NCRT and surgical resection between January 2009 and December 2014. Patients who did not undergo MRI before or after NCRT $(n=216)$ and those who did not undergo TME $(n=24)$ were excluded (S1 Fig.). Finally, 524 patients were included in the analyses.

\section{Treatments}

The clinical target volumes (CTVs) were defined as the volumes expanded from gross tumor volumes of $0.5-1.0 \mathrm{~cm}$ and regional lymphatic areas including presacral, internal iliac, obturator, and mesorectal areas. The CTV included the external iliac or inguinal area if there were lymph node metastases in those areas. The planning target volume (PTV) was delineated by expanding the CTV by $1.0 \mathrm{~cm}$. The beam margin was $0.5 \mathrm{~cm}$ around the PTV with adjustment according to the anatomic structures. The median dose of NCRT was $44.0 \mathrm{~Gy}$ (range, 33.0 to $60.0 \mathrm{~Gy}$ ) in a median of 22 fractions (range, 10 to 30 fractions) with daily doses per fraction, 1.8 to $3.3 \mathrm{~Gy}$. For some patients $(\mathrm{n}=113)$ with lateral lymph node metastasis or circumferential margin threatened in initial MRI, additional boost dose with 10-16 Gy in 5-8 fractions were applied to the lateral lymph node or tumor threatening the circumferential margin after the whole pelvic irradiation of $44.0 \mathrm{~Gy}$ in 22 fractions, according to the clinician's decision. Otherwise, NCRT to whole pelvis of 33.0 Gy in 10 fractions ( $\mathrm{n}=20), 44.0$ Gy in 22 fractions $(\mathrm{n}=386)$, or 50.4 Gy in 28 fractions $(n=5)$ were performed. Three-dimensional conformal radiotherapies were utilized. The chemotherapies consisted of 5-fluorouracil with intravenous bolus infusion ( $n=97)$, capecitabine $(n=262)$, 5-fluorouracil plus leucovorin with intra-venous infusion $(n=157)$, and others $(n=8)$. Adjuvant chemotherapies were administered in 482 patients.

\section{Pathologic examination}

The pathological staging was based on the 7th edition of the American Joint Committee on Cancer guidelines [20,21]. The tumor response grade (TRG) was assessed according to the Dworak's TRG system [21]. The pathological TRGs (pTRGs) were as follows: grade 0 , no response; grade 1, dominant tumor mass with obvious fibrosis, vasculopathy, or both (minimal response); grade 2, dominant fibrotic changes with a few easy-to-find tumor cells or groups (moderate response); grade 3, few (difficult to find microscopically) tumor cells in fibrotic tissue with or without mucous substance (near complete response); and grade 4, no viable tumor (complete response). The pTRGs were available in 514 patients. The circumferential resection margin was also evaluated according to the definition as shorter than $1 \mathrm{~mm}$ between the tumor and resection margin. The pTRGs were categorized into the good and the poor pTRG for binary comparison. The good and poor pTRGs were defined as pTRG3 or 4, and pTRG0, 1, or 2, respectively. 


\section{MRI examination}

All patients underwent pre-NCRT rectum MRI within 4 weeks before NCRT. The post-NCRT rectum MRIs were obtained at 6-8 weeks after completion of NCRT. Our routine protocol and sequence parameters of MRI are summarized in a previous report [22]. The tumor and nodal stages and mesorectal fascia involvement on MRI (mrMFI) before NCRT were assessed. mrMFI was defined if the distance between the tumor and the mesorectal fascia or levator muscle was less than $1 \mathrm{~mm}$ on MRI or there was the invasion to the intersphincteric plane or beyond. MRI assessment of TRG (mrTRG) was performed by a radiologist (K.D.S.) with 15 years of experience in MRI staging of rectal cancers. The mrTRG is based on a system analogous to the Dworak's pTRG system. The mrTRGs were as follows: grade 1 , no evidence of treated tumor; grade 2, dense hypointense fibrosis (minimal residual tumor); grade 3, $\sim 50 \%$ fibrosis / mucin and intermediate signal representing residual tumor; grade 4 , minimal fibrosis / mucinous degeneration, mostly tumor; and grade 5 , tumor has the same appearance as that at baseline. The examples of mrTRG were shown in the S2 Fig. As the pTRG, mrTRGs were classified into the good and the poor mrTRG for simplification of analysis. The good and poor mrTRGs included mrTRG1 or 2, and mrTRG3, 4, or 5, respectively.

\section{CEA parameters}

The initial CEA (iCEA) was evaluated within 4 weeks before the start of NCRT. The post-NCRT CEA was measured between 6 and 8 weeks after the completion of NCRT. The CEA parameters included the (1) iCEA with cut-off value identified based on receiver-operating characteristic (ROC) analysis for the highest accuracy in distinguishing the good pTRG from poor pTRG and (2) CEA dynamics by which patients were divided into three groups as follows: group 1, CEA level lower than the CEA cut-off value before and after NCRT; group 2, CEA level higher than the CEA cut-off value before NCRT, but lower after NCRT; and group 3, CEA level higher than the CEA cut-off value after NCRT.

\section{Statistical analysis}

The areas under the curves based on ROC analyses of mrTRG alone and the combination of mrTRG plus CEA parameters for the prediction of $\mathrm{pTRG}$ or $\mathrm{pCR}$ were compared using DeLong's method. The ROC analyses were performed using R Statistical Software ver. 3.2.3 (The R Foundation for Statistical Analyses, Vienna, Austria). Positive predictive values (PPVs) of good mrTRG according to the interval of iCEA were also investigated. Logistic regression was performed in univariate and multivariate analysis to identify the factors associated with good pTRG.

Overall survival (OS), DFS, and intrapelvic control were defined as the interval from the start date of NCRT to death, any cancer recurrence, and intrapelvic recurrence, respectively, or the last visit. The Kaplan-Meier method was used to estimate survival curves. Log-rank tests were performed to compare survival curves for various variables. Cox regression analysis was chosen for multivariate analysis to identify the independent prognostic factors for outcomes. A twosided $p$-value of $<0.05$ was considered statistically significant. The subgroup analyses were performed with stratification by MRI parameters identified as significant in multivariate analyses for DFS to evaluate whether the CEA para-

Table 1. Patient characteristics

\begin{tabular}{|c|c|}
\hline Characteristic & No. $(\%)(n=524)$ \\
\hline \multicolumn{2}{|l|}{ Sex } \\
\hline Male & $349(66.6)$ \\
\hline Female & $175(33.4)$ \\
\hline \multicolumn{2}{|l|}{ Histology } \\
\hline Adenocarcinoma & $507(96.8)$ \\
\hline Mucinous adenocarcinoma & $13(2.5)$ \\
\hline Signet ring cell & $4(0.7)$ \\
\hline \multicolumn{2}{|l|}{ Clinical T category } \\
\hline 1 & $4(0.8)$ \\
\hline 2 & 75 (14.3) \\
\hline 3 & $425(81.1)$ \\
\hline 4 & $20(3.8)$ \\
\hline \multicolumn{2}{|l|}{ Clinical N category } \\
\hline 0 & $36(6.9)$ \\
\hline 1 & $183(34.9)$ \\
\hline 2 & $305(58.2)$ \\
\hline \multicolumn{2}{|l|}{ Metastasis } \\
\hline Non-regional lymph node & $24(4.6)$ \\
\hline Liver & $7(1.3)$ \\
\hline Lung & $7(1.3)$ \\
\hline \multicolumn{2}{|l|}{ Operation } \\
\hline Low anterior resection & $470(89.7)$ \\
\hline Abdominoperineal resection & $44(8.4)$ \\
\hline Others & $10(1.9)$ \\
\hline \multicolumn{2}{|l|}{ iCEA (ng/mL) } \\
\hline$\leq 3.0$ & $306(58.4)$ \\
\hline$>3.0$ & $211(40.3)$ \\
\hline NA & $7(1.3)$ \\
\hline \multicolumn{2}{|l|}{ mrMFI } \\
\hline Yes & $210(40.1)$ \\
\hline No & $314(59.9)$ \\
\hline
\end{tabular}

iCEA, initial carcinomembryonic antigen; NA, not applicable; mrMFI, mesorectal fascia invasion on initial magnetic resonance imaging. 
meter could result in further discrimination of DFS. Statistical analyses for logistic regression, survival curves and prognosis factors were performed using SPSS ver. 22.0 (IBM Corp., Armonk, NY).

\section{Ethical statement}

This study was approved by the Institutional Review Board of Samsung Medical Center (SMC 2018-12-006-001). The waiver of informed consent was approved.

\section{Results}

\section{Patient characteristics}

Patient characteristics are summarized in Table 1 . The median age was 56 years (range, 27 to 82 years). The median iCEA was $2.6 \mathrm{ng} / \mathrm{mL}$ (range, 0.5 to $312.2 \mathrm{ng} / \mathrm{mL}$ ). The proportion of patients with iCEA less than or equivalent to 3 $\mathrm{ng} / \mathrm{mL}$, which was identified as the cut-off value for the highest accuracy in distinguishing the good pTRG from poor pTRG in ROC analysis, was 58.4\% (Table 1).

\section{Tumor responses and performance of mrTRG and CEA parameters in the prediction of pathologic response}

The tumor characteristics after NCRT are summarized in

Table 2. pTRG according to $\operatorname{mrTRG}(\mathrm{n}=514)$

\begin{tabular}{|c|c|c|c|c|c|c|}
\hline & \multicolumn{5}{|c|}{ pTRG } & \multirow{2}{*}{ Total } \\
\hline & 0 & 1 & 2 & 3 & 4 & \\
\hline mrTRG 1 & 0 & $8(6.6)$ & $36(29.8)$ & $25(20.7)$ & $52(43.0)$ & $121(23.5)$ \\
\hline mrTRG 2 & $1(0.5)$ & $18(9.7)$ & $82(44.3)$ & $45(24.3)$ & 39 (21.1) & $185(36.0)$ \\
\hline mrTRG 3 & $1(0.8)$ & $35(29.2)$ & $68(56.7)$ & $10(8.3)$ & $6(5.0)$ & $120(23.3)$ \\
\hline mrTRG 4 & $2(2.7)$ & $21(28.8)$ & $39(53.4)$ & $6(8.2)$ & $5(6.8)$ & 73 (14.2) \\
\hline mrTRG 5 & $1(6.7)$ & 8 (53.3) & $5(33.3)$ & 0 & $1(6.7)$ & $15(2.9)$ \\
\hline Total & $5(1.0)$ & 90 (17.5) & $230(44.7)$ & 86 (16.7) & $103(20.0)$ & $514(100)$ \\
\hline
\end{tabular}

Values are presented as number $(\%)$. pTRG, pathologic tumor regression grade; mrTRG, magnetic resonance imaging assessment of tumor regression grade.

Table 3. Characteristics associated with pTRG (poor vs. good; $\mathrm{n}=514$ )

\begin{tabular}{|c|c|c|c|c|}
\hline \multirow{2}{*}{ Characteristic } & \multicolumn{2}{|c|}{ Univariate analysis } & \multicolumn{2}{|c|}{ Multivariate analysis } \\
\hline & OR $(95 \% \mathrm{CI})$ & p-value & OR $(95 \% \mathrm{CI})$ & p-value \\
\hline \multicolumn{5}{|l|}{ cT category } \\
\hline 1,2 vs. 3,4 & $0.478(0.295-0.775)$ & 0.003 & $0.807(0.467-1.393)$ & 0.441 \\
\hline \multicolumn{5}{|l|}{ cN category } \\
\hline 0, 1 vs. 2 & $1.002(0.697-1.441)$ & 0.992 & $1.387(0.908-2.120)$ & 0.130 \\
\hline \multicolumn{5}{|l|}{ mrTRG } \\
\hline 1-2 vs. $3-5$ & $0.140(0.089-0.221)$ & $<0.001$ & $0.171(0.106-0.275)$ & $<0.001$ \\
\hline \multicolumn{5}{|l|}{ mrMFI } \\
\hline No vs. Yes & $0.497(0.340-0.728)$ & $<0.001$ & $0.658(0.426-1.016)$ & 0.059 \\
\hline \multicolumn{5}{|l|}{ iCEA (ng/mL) } \\
\hline$\leq 3.0$ vs. $>3.0$ & $0.297(0.199-0.444)$ & $<0.001$ & $0.382(0.247-0.589)$ & $<0.001$ \\
\hline
\end{tabular}

pTRG, pathologic tumor regression grade; OR, odds ratio; $\mathrm{CI}$, confidential interval; mrTRG, magnetic resonance imaging assessment of tumor regression grade; mrMFI, mesorectal fascia invasion on initial magnetic resonance imaging; iCEA, initial carcinoembryonal antigen. 


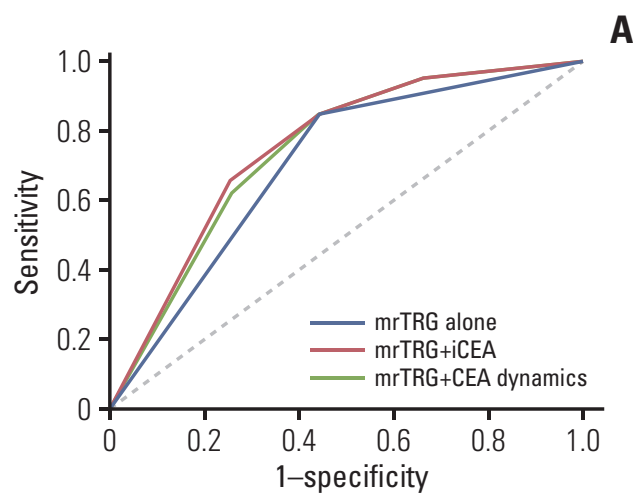

A

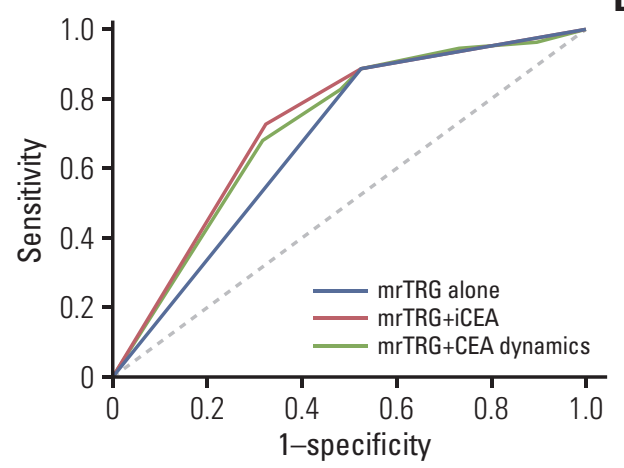

B

\begin{tabular}{lccccc}
\multirow{2}{*}{ Parameter } & \multicolumn{2}{c}{ pTRG3-4 (good pTRG) } & & \multicolumn{2}{c}{ pTRG4 (pCR) } \\
\cline { 2 - 3 } \cline { 5 - 6 } & AUC $(95 \%$ CI $)$ & p-value & & AUC (95\% CI) & p-value -val $^{\text {a) }}$ \\
mrTRG alone & $0.703(0.666-0.740)$ & - & & $0.680(0.280-0.359)$ & - \\
mrTRG+iCEA & $0.751(0.711-0.791)$ & $<0.001$ & & $0.728(0.681-0.774)$ & $<0.001$ \\
mrTRG+CEA dynamics & $0.743(0.703-0.784)$ & $<0.001$ & & $0.715(0.666-0.764)$ & $<0.001$ \\
\hline
\end{tabular}

Fig. 1. Receiver-operating characteristic curves of tumor regression grade on magnetic resonance imaging (mrTRG) (good vs. poor) alone and the combination of mrTRG plus carcinoembryonic antigen (CEA) parameters (initial CEA [iCEA] and CEA dynamics) for the prediction of pathologic tumor regression grade ( $p$ TRG) (A) and pathologic complete response (pCR) (B). CI, confidence interval. a) $\mathrm{p}$-values are for the comparison of each area under the curve (AUC) and that of mrTRG based on DeLong's method.
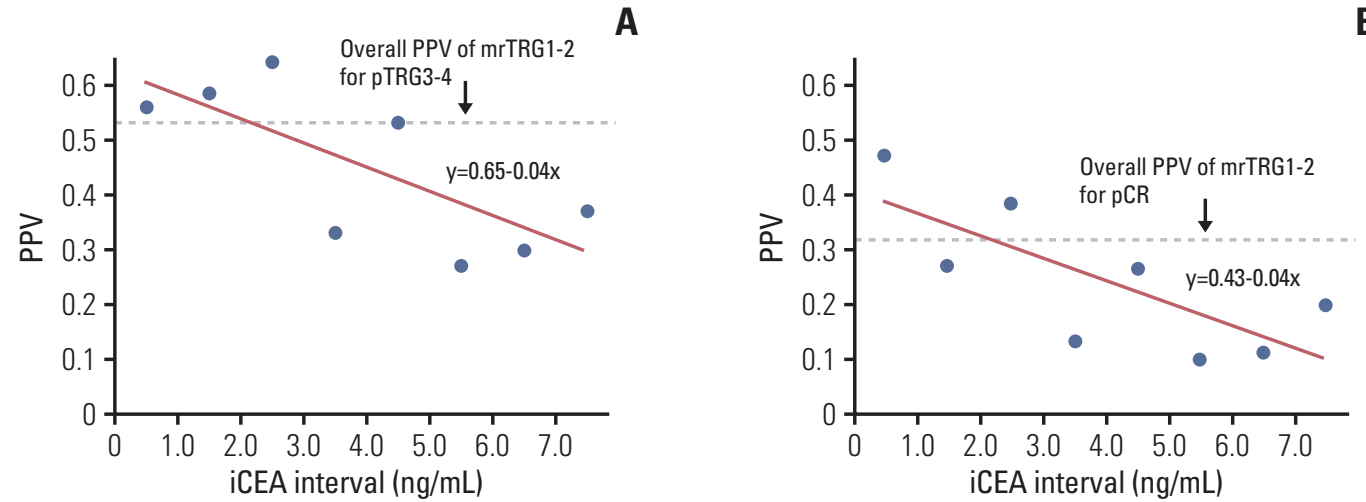

Fig. 2. The positive predictive values (PPVs) of good tumor regression grade on magnetic resonance imaging (mrTRG) for good pathologic tumor regression grade (pTRG) (A) and pathologic complete response (pCR) (B) according to the interval of initial carcinoembryonic antigen (iCEA).

S3 Table. The median CEA after NCRT was $1.6 \mathrm{ng} / \mathrm{mL}$ (range, 0.1 to $40.3 \mathrm{ng} / \mathrm{mL}$ ). Of the patients with $\mathrm{iCEA}>3$ $\mathrm{ng} / \mathrm{mL}$, the median CEA changed from $5.81 \mathrm{ng} / \mathrm{mL}$ (range, 3.0 to $312.2 \mathrm{ng} / \mathrm{mL}$ ) to $2.28 \mathrm{ng} / \mathrm{mL}$ (range, 0.5 to $40.3 \mathrm{ng} / \mathrm{mL}$ ) after NCRT. T down-staging was achieved in 260 patients (49.6\%). The pTRGs according to each mrTRG in 514 patients whose pTRGs were available are shown in Table 2. Good
pTRG and pCR were shown in 189 (36.7\%) and $103(20.0 \%)$ patients, respectively. In the multivariate analysis, good mrTRG $(p<0.001)$ and iCEA of 3.0 or less $(p<0.001)$ were identified as independent factors associated with good pTRG (Table 3). The mrMFI showed marginal significance in the association with good pTRG $(\mathrm{p}=0.059)$ (Table 3$)$.

Fig. 1 shows the ROC curves of mrTRG (good vs. poor) 
Table 4. Univariate and multivariate analysis for DFS

\begin{tabular}{|c|c|c|c|c|}
\hline \multirow{2}{*}{ Variable } & \multicolumn{2}{|c|}{ Univariate analysis } & \multicolumn{2}{|c|}{ Multivariate analysis } \\
\hline & 5-Year DFS rate $(\%)$ & p-value & HR $(95 \%$ CI $)$ & p-value \\
\hline \multicolumn{5}{|c|}{ cT category } \\
\hline 1,2 & 73.1 & 0.652 & $0.708(0.421-1.193)$ & 0.195 \\
\hline 3,4 & 75.0 & & & \\
\hline \multicolumn{5}{|c|}{$\mathrm{cN}$ category } \\
\hline 0,1 & 77.4 & 0.201 & $0.089(0.620-1.302)$ & 0.570 \\
\hline 2 & 72.8 & & & \\
\hline \multicolumn{5}{|l|}{ mrTRG } \\
\hline $1-2$ & 78.6 & 0.007 & $0.687(0.473-0.997)$ & 0.048 \\
\hline $3-5$ & 68.9 & & & \\
\hline \multicolumn{5}{|l|}{ mrMFI } \\
\hline No & 79.8 & 0.003 & $0.662(0.461-0.951)$ & 0.026 \\
\hline Yes & 67.2 & & & \\
\hline \multicolumn{5}{|c|}{ iCEA (ng/mL) } \\
\hline$\leq 3.0$ & 79.1 & 0.003 & $0.675(0.471-0.966)$ & 0.032 \\
\hline$>3.0$ & 69.1 & & & \\
\hline
\end{tabular}

DFS, disease-free survival; HR, hazard ratio; CI, confidential interval; mrTRG, magnetic resonance imaging assessment of tumor regression grade; $\mathrm{mrMFI}$, mesorectal fascia invasion on initial magnetic resonance imaging; iCEA, initial carcinoembryonal antigen.

alone and the combination of mrTRG plus CEA parameters (iCEA and CEA dynamics) for the prediction of pTRG and $\mathrm{pCR}$. The areas under the curves were significantly larger in mrTRG plus CEA parameters than in mrTRG alone for the prediction of both pTRG and pCR (Fig. 1). The PPVs of good mrTRG for good pTRG and pCR showed tendencies to decrease according to the increase in iCEA (Fig. 2).

\section{Survival analyses}

The median follow-up was 64.0 months (range, 3.0 to 113.0 months). The actuarial rates of intrapelvic control, DFS, and OS at 5 years were $89.5 \%, 77.5 \%$, and $88.3 \%$, respectively (S4 Fig.). The univariate and multivariate analyses for DFS and OS are shown in Table 4 and S5 Table, respectively. On the multivariate analysis, poor mrTRG, iCEA $>3.0 \mathrm{ng} / \mathrm{mL}$, and mrMFI before NCRT, which were identified as factors associated with poor pTRG, were also independent risk factors associated with poor DFS (Table 4). In the subgroup analyses, the iCEA discriminated the DFS significantly in the subgroup with good mrTRG $(\mathrm{p}=0.012)$ or that without mrMFI (p=0.004) (Fig. 3).

\section{Discussion}

The reliable prediction of pathologic response is essential for the selection of patients with rectal cancer whose surgery can be omitted safely after NCRT. Various parameters including mrTRG and tumor markers have been identified to be correlated with pathologic response. However, it is insufficient to use any specific parameters as surrogates of pathologic response because of the unsatisfactory performance. To improve the performance, a combination of multiple parameters is a plausible approach. To our knowledge, this study is the first study investigating whether the combination of MRI parameters and CEA levels compared to MRI parameters alone show improved performance in the prediction of pathologic response after NCRT for rectal cancer.

The present results showed that the iCEA and CEA dynamics after NCRT significantly improved the performance of mrTRG in the prediction of pTRG. This implied that the complex evaluation of multiple preoperative parameters can predict pathologic response better than the evaluation of single parameters. In addition, the correlation of increased iCEA with lowered PPV of good mrTRG in the prediction of good pTRG and pCR also connote that iCEA has the potential to be utilized in the screening of false positivity of good mrTRG for good pTRG or pCR. For a successful watch-and-wait policy, the exclusion of patients who should not omit surgery 
A
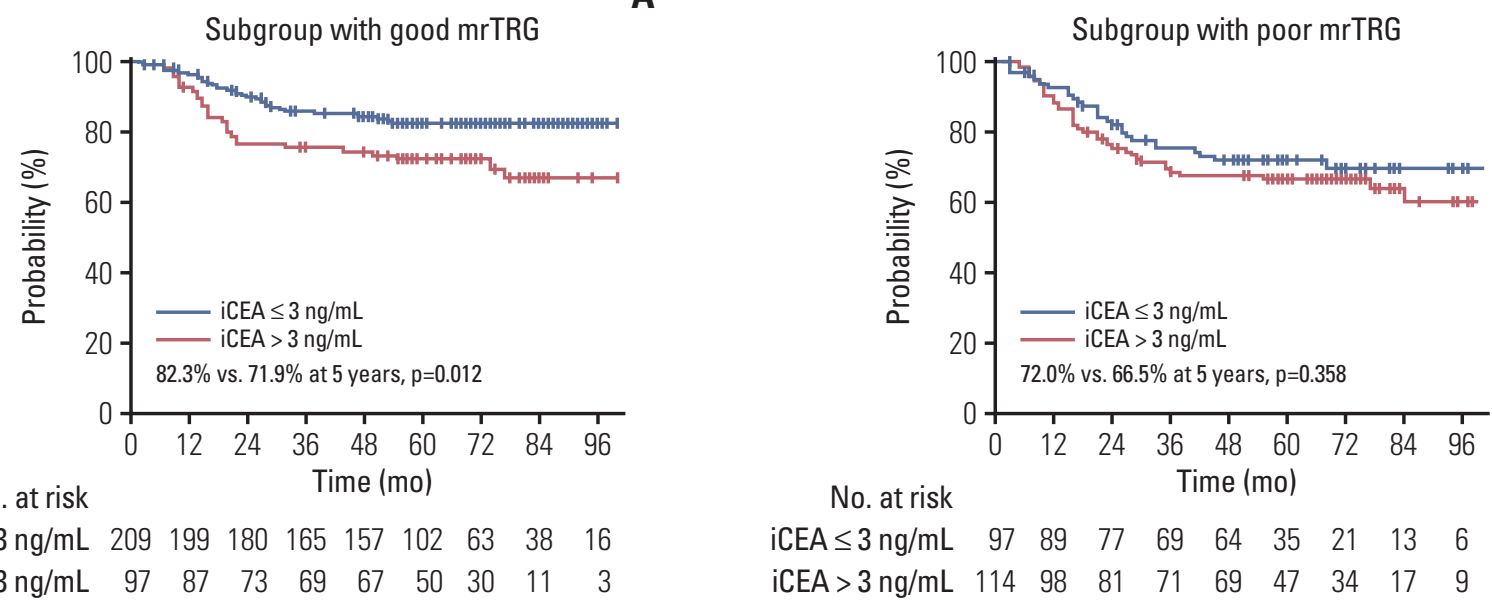

$\begin{array}{lrrrrrrrrr}\text { iCEA } \leq 3 \mathrm{ng} / \mathrm{mL} & 209 & 199 & 180 & 165 & 157 & 102 & 63 & 38 & 16 \\ \text { iCEA }>3 \mathrm{ng} / \mathrm{mL} & 97 & 87 & 73 & 69 & 67 & 50 & 30 & 11 & 3\end{array}$

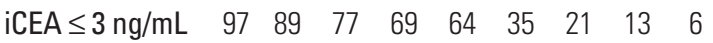

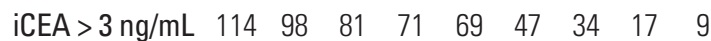
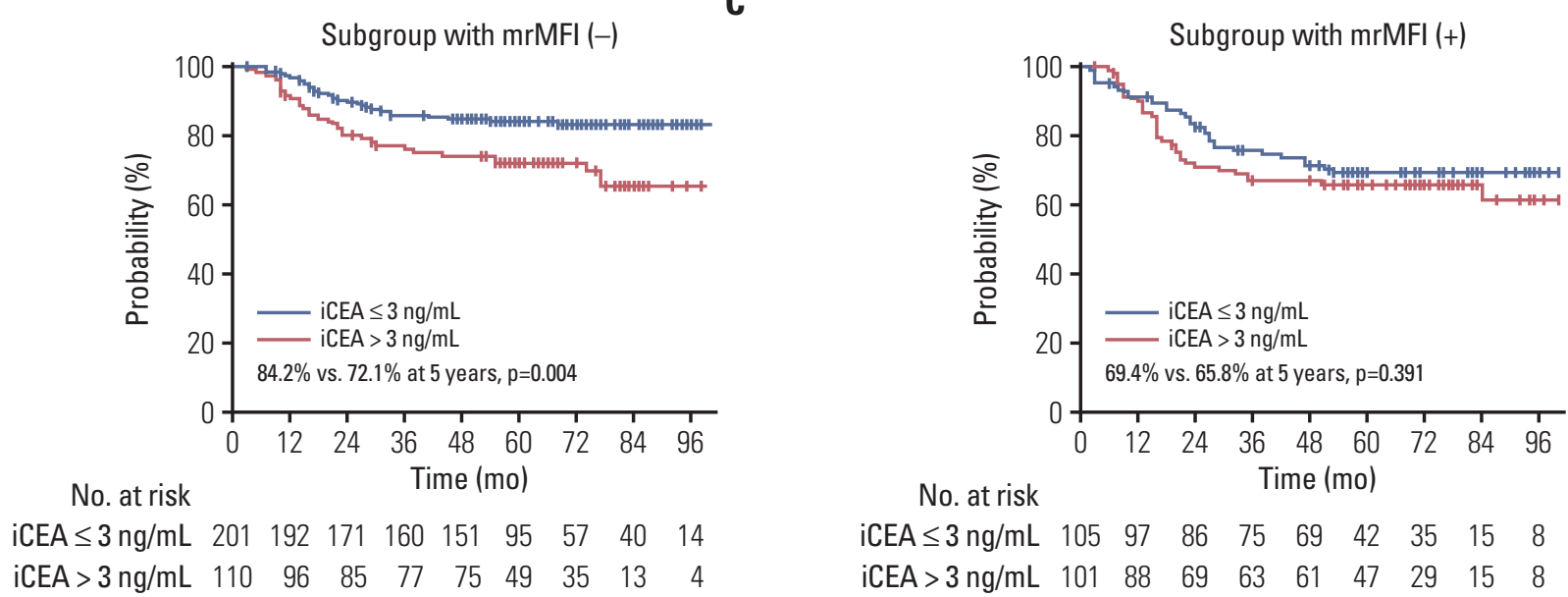

Fig. 3. Disease-free survival of the subgroups with good tumor regression grade on magnetic resonance imaging (mrTRG) (A), poor mrTRG (B), negative mesorectal fascia invasion on initial magnetic resonance imaging (mrMFI) (C), and positive mrMFI (D). iCEA, initial carcinoembryonic antigen.

even with good clinical and radiologic response, as well as the inclusion of those who can omit surgery safely, is essential. Therefore, increased iCEA can be a good parameter in the selection of patients with good mrTRG unsuitable for the watch-and-wait approach because of its implication of lowered PPV of good mrTRG.

Despite the significant improvement in the performance of MRI parameters in the prediction of pTRG by the combination with iCEA, the performance is still not satisfactory with areas under the curve of 0.715-0.751, which is the level of moderate predictivity [23]. For further improvement of the performance, additional radiologic parameters are considerable to be utilized. Several reports have reported the application of diffusion-weighted imaging (DWI) parameters and dynamic contrast-enhanced MRI in the prediction of patho- logic response after NCRT for rectal cancer [24-26]. In particular, Bulens et al. [24] built a model with T2-volumetric and DWI parameters and showed its good predictive performance for good pTRG (area under the curve, 0.89 ; 95\% confidential interval, 0.79 to 0.98 ). The combination of ${ }^{18} \mathrm{~F}$-fluorodeoxyglucose positron emission tomography $\left({ }^{18} \mathrm{~F}\right.$-FDG PET) parameters is also considerable. Various studies have investigated the values of ${ }^{18} \mathrm{~F}$-FDG PET parameters, such as maximum standardized uptake values or their dynamics before and after NCRT [22,27-32]. However, the applications of ${ }^{18}$ F-FDG PET parameters or their combination with MRI parameters remain controversial [33], and further investigations are required.

For the adequate watch-and-wait approach, the selection of patients with a low risk of any recurrence, as well as 
potential pCR, is also important. In the present study, mrMFI before NCRT was identified as an independent prognostic factor associated with DFS, in addition to mrTRG and iCEA. The mrMFI has been identified as a risk factor in previous studies $[34,35]$. In particular, the MERCURY study showed that mrMFI was significantly associated with distant metastasis and suggested intensified treatment for patients with rectal cancer with mrMFI [34]. Therefore, the omission of surgery should be carefully determined for patients with rectal cancer with mrMFI, even if iCEA is low and good mrTRG is achieved. In addition, our study revealed that in subgroup with good mrTRG or that without mrMFI, iCEA discriminated the DFS significantly, implying that iCEA could be a tool of selection for patients with significantly shorter DFS even in patients with favorable MRI findings. While the DFS rates at 5 years were over $82 \%$ in those subgroups with iCEA $\leq 3 \mathrm{ng} / \mathrm{mL}$, the rates declined to around $72 \%$ in those subgroups with iCEA $>3 \mathrm{ng} / \mathrm{mL}$. Therefore, the watch-and-wait approach needs to be restricted in patients with high iCEA even if there is no mrMFI on initial MRI or good mrTRG. Further studies are necessary to determine the subgroup of patients appropriate for the watch-and-wait approach.

There are several limitations in the present study. This study is a retrospective study, which has inevitable selection bias. In addition, it is insufficient to regard the MRI findings reviewed by only one radiologist in the present study universally consistent because there could be discrepancies in the evaluation of mrTRG among the radiologists $[15,22]$. Therefore, a large-scaled prospective study with a well-organized review of images and pathology is required to confirm the result.

In conclusion, iCEA and CEA dynamics after NCRT significantly improve the performance of mrTRG in the prediction of pTRG. The increase in iCEA correlates the lowered PPV of good mrTRG in the prediction of good pTRG. The iCEA also discriminates DFS significantly in the subgroups with favorable MRI parameters such as good mrTRG and negative mrMFI.

\section{Electronic Supplementary Material}

Supplementary materials are available at Cancer Research and Treatment website (https: // www.e-crt.org).

\section{Conflicts of Interest}

Conflict of interest relevant to this article was not reported.

\section{References}

1. Sauer R, Becker H, Hohenberger W, Rodel C, Wittekind C, Fietkau R, et al. Preoperative versus postoperative chemoradiotherapy for rectal cancer. N Engl J Med. 2004;351:1731-40.

2. Song JH, Jeong JU, Lee JH, Kim SH, Cho HM, Um JW, et al. Preoperative chemoradiotherapy versus postoperative chemoradiotherapy for stage II-III resectable rectal cancer: a metaanalysis of randomized controlled trials. Radiat Oncol J. 2017;35:198-207.

3. Rodel C, Martus P, Papadoupolos T, Fuzesi L, Klimpfinger M, Fietkau R, et al. Prognostic significance of tumor regression after preoperative chemoradiotherapy for rectal cancer. J Clin Oncol. 2005;23:8688-96.

4. Losi L, Luppi G, Gavioli M, Iachetta F, Bertolini F, D'Amico R, et al. Prognostic value of Dworak grade of regression (GR) in patients with rectal carcinoma treated with preoperative radiochemotherapy. Int J Colorectal Dis. 2006;21:645-51.

5. Maas M, Nelemans PJ, Valentini V, Das P, Rodel C, Kuo LJ, et al. Long-term outcome in patients with a pathological complete response after chemoradiation for rectal cancer: a pooled analysis of individual patient data. Lancet Oncol. 2010;11:83544.

6. Capirci C, Valentini V, Cionini L, De Paoli A, Rodel C, GlynneJones R, et al. Prognostic value of pathologic complete res- ponse after neoadjuvant therapy in locally advanced rectal cancer: long-term analysis of 566 ypCR patients. Int J Radiat Oncol Biol Phys. 2008;72:99-107.

7. Kennedy GD. Hot topics in colorectal surgery. Clin Colon Rectal Surg. 2016;29:183-4.

8. Huang M, Lin J, Yu X, Chen S, Kang L, Deng Y, et al. Erectile and urinary function in men with rectal cancer treated by neoadjuvant chemoradiotherapy and neoadjuvant chemotherapy alone: a randomized trial report. Int J Colorectal Dis. 2016;31:1349-57.

9. Park SY, Choi GS, Park JS, Kim HJ, Ryuk JP, Yun SH. Urinary and erectile function in men after total mesorectal excision by laparoscopic or robot-assisted methods for the treatment of rectal cancer: a case-matched comparison. World J Surg. 2014; 38:1834-42.

10. van der Valk MJM, Hilling DE, Bastiaannet E, MeershoekKlein Kranenbarg E, Beets GL, Figueiredo NL, et al. Long-term outcomes of clinical complete responders after neoadjuvant treatment for rectal cancer in the International Watch \& Wait Database (IWWD): an international multicentre registry study. Lancet. 2018;391:2537-45.

11. Dossa F, Chesney TR, Acuna SA, Baxter NN. A watch-andwait approach for locally advanced rectal cancer after a clinical 
complete response following neoadjuvant chemoradiation: a systematic review and meta-analysis. Lancet Gastroenterol Hepatol. 2017;2:501-13.

12. Renehan AG, Malcomson L, Emsley R, Gollins S, Maw A, Myint AS, et al. Watch-and-wait approach versus surgical resection after chemoradiotherapy for patients with rectal cancer (the OnCoRe project): a propensity-score matched cohort analysis. Lancet Oncol. 2016;17:174-83.

13. Kim JH. Controversial issues in radiotherapy for rectal cancer: a systematic review. Radiat Oncol J. 2017;35:295-305.

14. Goodman KA. Definitive chemoradiotherapy ("Watch-andWait" approach). Semin Radiat Oncol. 2016;26:205-10.

15. Patel UB, Taylor F, Blomqvist L, George C, Evans H, Tekkis P, et al. Magnetic resonance imaging-detected tumor response for locally advanced rectal cancer predicts survival outcomes: MERCURY experience. J Clin Oncol. 2011;29:3753-60.

16. Patel UB, Brown G, Rutten H, West N, Sebag-Montefiore D, Glynne-Jones R, et al. Comparison of magnetic resonance imaging and histopathological response to chemoradiotherapy in locally advanced rectal cancer. Ann Surg Oncol. 2012; 19:2842-52.

17. Lee JH, Kim DY, Kim SH, Cho HM, Shim BY, Kim TH, et al. Carcinoembryonic antigen has prognostic value for tumor downstaging and recurrence in rectal cancer after preoperative chemoradiotherapy and curative surgery: a multi-institutional and case-matched control study of KROG 14-12. Radiother Oncol. 2015;116:202-8.

18. Kim CW, Yu CS, Yang SS, Kim KH, Yoon YS, Yoon SN, et al. Clinical significance of pre- to post-chemoradiotherapy s-CEA reduction ratio in rectal cancer patients treated with preoperative chemoradiotherapy and curative resection. Ann Surg Oncol. 2011;18:3271-7.

19. Chung MJ, Nam TK, Jeong JU, Kim SH, Kim K, Jang HS, et al. Can serum dynamics of carcinoembryonic antigen level during neoadjuvant chemoradiotherapy in rectal cancer predict tumor response and recurrence? A multi-institutional retrospective study. Int J Colorectal Dis. 2016;31:1595-601.

20. Edge SB, Compton CC. The American Joint Committee on Cancer: the 7th edition of the AJCC cancer staging manual and the future of TNM. Ann Surg Oncol. 2010;17:1471-4.

21. Dworak O, Keilholz L, Hoffmann A. Pathological features of rectal cancer after preoperative radiochemotherapy. Int J Colorectal Dis. 1997;12:19-23.

22. Song I, Kim SH, Lee SJ, Choi JY, Kim MJ, Rhim H. Value of diffusion-weighted imaging in the detection of viable tumour after neoadjuvant chemoradiation therapy in patients with locally advanced rectal cancer: comparison with $\mathrm{T} 2$ weighted and PET/CT imaging. Br J Radiol. 2012;85:577-86.

23. Greiner M, Pfeiffer D, Smith RD. Principles and practical application of the receiver-operating characteristic analysis for diagnostic tests. Prev Vet Med. 2000;45:23-41.

24. Bulens P, Couwenberg A, Haustermans K, Debucquoy A, Vandecaveye V, Philippens $M$, et al. Development and validation of an MRI-based model to predict response to chemoradiotherapy for rectal cancer. Radiother Oncol. 2018;126:437-42.
25. Lambrecht M, Vandecaveye V, De Keyzer F, Roels S, Penninckx F, Van Cutsem E, et al. Value of diffusion-weighted magnetic resonance imaging for prediction and early assessment of response to neoadjuvant radiochemotherapy in rectal cancer: preliminary results. Int J Radiat Oncol Biol Phys. 2012;82:863-70.

26. Barbaro B, Vitale R, Valentini V, Illuminati S, Vecchio FM, Rizzo G, et al. Diffusion-weighted magnetic resonance imaging in monitoring rectal cancer response to neoadjuvant chemoradiotherapy. Int J Radiat Oncol Biol Phys. 2012;83: 594-9.

27. Joye I, Deroose CM, Vandecaveye V, Haustermans K. The role of diffusion-weighted MRI and (18)F-FDG PET / CT in the prediction of pathologic complete response after radiochemotherapy for rectal cancer: a systematic review. Radiother Oncol. 2014;113:158-65.

28. Lee SJ, Kim JG, Lee SW, Chae YS, Kang BW, Lee YJ, et al. Clinical implications of initial FDG-PET/CT in locally advanced rectal cancer treated with neoadjuvant chemoradiotherapy. Cancer Chemother Pharmacol. 2013;71:1201-7.

29. Hindie E, Hennequin C, Moretti JL. Predicting response to chemoradiotherapy in rectal and oesophageal cancer with $18 \mathrm{~F}$ FDG: prognostic value and possible role in patient management. Eur J Nucl Med Mol Imaging. 2007;34:1576-82.

30. Leccisotti L, Gambacorta MA, de Waure C, Stefanelli A, Barbaro B, Vecchio FM, et al. The predictive value of 18F-FDG $\mathrm{PET} / \mathrm{CT}$ for assessing pathological response and survival in locally advanced rectal cancer after neoadjuvant radiochemotherapy. Eur J Nucl Med Mol Imaging. 2015;42:657-66.

31. Murcia Durendez MJ, Frutos Esteban L, Lujan J, Frutos MD, Valero G, Navarro Fernandez JL, et al. The value of 18F-FDG $\mathrm{PET} / \mathrm{CT}$ for assessing the response to neoadjuvant therapy in locally advanced rectal cancer. Eur J Nucl Med Mol Imaging. 2013;40:91-7.

32. Koc M, Kaya GC, Demir Y, Surucu E, Sarioglu S, Obuz F, et al. The value of liver-based standardized uptake value and other quantitative 18F-FDG PET-CT parameters in neoadjuvant therapy response in patients with locally advanced rectal cancer: correlation with histopathology. Nucl Med Commun. 2015;36:898-907.

33. Aiba T, Uehara K, Nihashi T, Tsuzuki T, Yatsuya H, Yoshioka $Y$, et al. MRI and FDG-PET for assessment of response to neoadjuvant chemotherapy in locally advanced rectal cancer. Ann Surg Oncol. 2014;21:1801-8.

34. Taylor FG, Quirke P, Heald RJ, Moran BJ, Blomqvist L, Swift $I R$, et al. Preoperative magnetic resonance imaging assessment of circumferential resection margin predicts disease-free survival and local recurrence: 5-year follow-up results of the MERCURY study. J Clin Oncol. 2014;32:34-43.

35. Kim H, Myoung S, Koom WS, Kim NK, Kim MJ, Ahn JB, et al. MRI risk stratification for tumor relapse in rectal cancer achieving pathological complete remission after neoadjuvant chemoradiation therapy and curative resection. PLoS One. 2016;11:e0146235. 